\title{
Intersectional soft sets theory applied to generalized hypervector spaces
}

\author{
G. Muhiuddin
}

\begin{abstract}
The notion of $\theta$-generalized int-soft subfields, $\theta$-generalized int-soft algebras over $\theta$-generalized int-soft subfields, and $\theta$-generalized int-soft hypervector spaces are introduced, and their properties and characterizations are considered. In connection with linear transformations, $\theta$ generalized int-soft hypervector spaces are discussed.
\end{abstract}

\section{Introduction}

The hyper-structure theory was introduced by Marty [33] at the 8th congress of Scandinavian Mathematicians in 1934. Since then many researchers have worked on hyperalgebraic structures and developed this theory (for more see [3], [4], [9], [10], [39]). In 1990, M. S. Tallini introduced the notion of hypervector spaces ([37], [38]) and studied basic properties of them. Molodtsov [34] introduced the concept of soft set as a new mathematical tool for dealing with uncertainties that is free from the difficulties that have troubled the usual theoretical approaches. Molodtsov pointed out several directions for the applications of soft sets. At present, works on the soft set theory are progressing rapidly. Maji et al. [32] described the application of soft set theory to a decision making problem. Maji et al. [31] also studied several operations on the theory of soft sets. Chen et al. [8] presented a new definition of

Key Words: $\theta$-generalized int-soft subfields, $\theta$-generalized int-soft algebras, $\theta$-generalized int-soft hypervector spaces.

2010 Mathematics Subject Classification: Primary 06D72, 20N20.

Received: 13-09-2019.

Accepted: 19-02-2020. 
soft set parametrization reduction, and compared this definition to the related concept of attributes reduction in rough set theory. The algebraic structure of set theories dealing with uncertainties has been studied by some authors. Feng [11] considered the application of soft rough approximations in multicriteria group decision making problems. Aktaş and Çağman [5] studied the basic concepts of soft set theory, and compared soft sets to fuzzy and rough sets, providing examples to clarify their differences. They also discussed the notion of soft groups. After than, many algebraic properties of soft sets are studied (see [1], [6], [12], [25], [26], [27], [28], [29], [35], [40]). Muhiuddin et al. studied the soft set theory on various aspects (see for e.g., [2], [13], [14], [15], [16], [17], [18], [36]). In recent years, a number of research papers have been devoted to the study of fuzzy sets theory and related concepts on different algebraic structures (see e.g., [19], [23], [24], [20], [21], [22]). In [30], Jun et al. introduced the notion of int-soft subfields, int-soft algebras over int-soft subfields, and int-soft hypervector spaces. They studied their properties and characterizations. In connection with linear transformations, they discussed int-soft hypervector spaces.

The aim of this paper is to consider generalizations of the paper [30]. We introduce the notion of $\theta$-generalized int-soft subfields, $\theta$-generalized int-soft algebras over $\theta$-generalized int-soft subfields, and $\theta$-generalized int-soft hypervector spaces. We study their properties and characterizations. In connection with linear transformations, we discusse $\theta$-generalized int-soft hypervector spaces.

\section{Preliminaries}

A soft set theory is introduced by Molodtsov [34], and Çağman et al. [7] provided new definitions and various results on soft set theory.

In what follows, let $U$ be an initial universe set and $E$ be a set of parameters. Let $\mathscr{P}(U)$ denotes the power set of $U, \mathscr{P}^{*}(U)=\mathscr{P}(U) \backslash\{\emptyset\}$ and $A, B, C, \cdots \subseteq E$.

A soft set $(\tilde{f}, A)$ over $U$ is defined to be the set of ordered pairs

$$
(\tilde{f}, A):=\{(x, \tilde{f}(x)): x \in E, \tilde{f}(x) \in \mathscr{P}(U)\},
$$

where $\tilde{f}: E \rightarrow \mathscr{P}(U)$ such that $\tilde{f}(x)=\emptyset$ if $x \notin A$.

For any sets $X$ and $Y$, let $\mu: X \rightarrow Y$ be a function and $(\tilde{f}, X)$ and $(\tilde{g}, Y)$ be soft sets over $U$.

(1) The soft set

$$
\mu^{-1}(\tilde{g}, Y)=\left\{\left(x, \mu^{-1}(\tilde{g})(x)\right): x \in X, \mu^{-1}(\tilde{g})(x) \in \mathscr{P}(U)\right\},
$$


where $\mu^{-1}(\tilde{g})(x)=\tilde{g}(\mu(x))$, is called the soft pre-image of $(\tilde{g}, Y)$ under $\mu$.

(2) The soft set

$$
\mu(\tilde{f}, X)=\{(y, \mu(\tilde{f})(y)): y \in Y, \mu(\tilde{f})(y) \in \mathscr{P}(U)\}
$$

where

$$
\mu(\tilde{f})(y)= \begin{cases}\bigcup_{x \in \mu^{-1}(y)} \tilde{f}(x) & \text { if } \mu^{-1}(y) \neq \emptyset \\ \emptyset & \text { otherwise }\end{cases}
$$

is called the soft image of $(\tilde{f}, X)$ under $\mu$.

A map $\circ: H \times H \rightarrow \mathscr{P}^{*}(H)$ is called a hyperoperation or join operation. The join operation is extended to subsets of $H$ in natural way, so that $A \circ B$ is given by

$$
A \circ B=\bigcup\{a \circ b \mid a \in A, b \in B\} .
$$

The notations $a \circ A$ and $A \circ a$ are used for $\{a\} \circ A$ and $A \circ\{a\}$ respectively. Generally, the singleton $\{a\}$ is identified by its element $a$.

Let $F$ be a field and $(V,+)$ be an abelian group. A hypervector space over $F$ (see [37]) is defined to be the quadruplet $(V,+, \circ, F)$, where "o" is a mapping

$$
\circ: F \times V \rightarrow \mathscr{P}^{*}(V)
$$

such that for all $a, b \in F$ and $x, y \in V$ the following conditions hold:

(H1) $a \circ(x+y) \subseteq a \circ x+a \circ y$,

(H2) $(a+b) \circ x \subseteq a \circ x+b \circ x$,

(H3) $a \circ(b \circ x)=(a b) \circ x$,

(H4) $a \circ(-x)=(-a) \circ x=-(a \circ x)$,

(H5) $x \in 1 \circ x$.

A hypervector space $(V,+, \circ, F)$ over a field $F$ is said to be strongly left distributive (see [3]) if it satisfies the following condition:

$$
(\forall a \in F)(\forall x, y \in V)(a \circ(x+y)=a \circ x+a \circ y)
$$

\section{Generalized int-soft algebras over an int-soft field}

In what follows let $F$ be a field unless otherwise specified. 
Definition 3.1 ([30]). A soft set $(\tilde{f}, F)$ over $F$ is called an int-soft subfield of $F$ if the following conditions are satisfied:

(1) $(\forall a, b \in F)(\tilde{f}(a+b) \supseteq \tilde{f}(a) \cap \tilde{f}(b))$.

(2) $(\forall a \in F)(\tilde{f}(-a) \supseteq \tilde{f}(a))$.

(3) $(\forall a, b \in F)(\tilde{f}(a) \cap \tilde{f}(b) \subseteq \tilde{f}(a b))$.

(4) $(\forall a \in F)\left(a \neq 0 \Rightarrow \tilde{f}(a) \subseteq \tilde{f}\left(a^{-1}\right)\right)$.

Definition 3.2. A soft set $(\tilde{f}, F)$ over $F$ is called a $\theta$-generalized int-soft subfield of $F$ over $U$ if there exists $\theta \in \mathscr{P}^{*}(U)$ such that

$$
\begin{aligned}
& (\forall a, b \in F)(\tilde{f}(a+b) \supseteq \theta \cap \tilde{f}(a) \cap \tilde{f}(b)) . \\
& (\forall a \in F)(\tilde{f}(-a) \supseteq \theta \cap \tilde{f}(a)) . \\
& (\forall a, b \in F)(\tilde{f}(a b) \supseteq \theta \cap \tilde{f}(a) \cap \tilde{f}(b)) . \\
& (\forall a \in F)\left(a \neq 0 \Rightarrow \tilde{f}\left(a^{-1}\right) \supseteq \theta \cap \tilde{f}(a)\right) .
\end{aligned}
$$

Note that the $U$-generalized int-soft subfield is only the int-soft subfield.

Example 3.3. (1) Let us consider $F=\left\{\mathbb{Z}_{3},+,.\right\}$ and $S=\mathbb{Z}_{3} \leq \mathbb{Z}_{3}$. Then the soft set $(\tilde{f}, S)$ is a soft set over $F$ where $\tilde{f}: S \rightarrow \mathscr{P}\left(\mathbb{Z}_{3}\right)$ such that $\tilde{f}(0)=\mathbb{Z}_{3}$ and $\tilde{f}(1)=\tilde{f}(2)=\{1,2\}$ which all are subfields of $F$. Hence, $(\tilde{f}, S)$ is a soft subfield over $F$.

(2) By Example $3.3(1)$, it is easy to verify that the soft set $(\tilde{f}, S)$ is an int-soft subfield over $F$. Furthermore, for any $\theta=\{1,2\} \in \mathscr{P}^{*}\left(\mathbb{Z}_{3}\right)$ the soft set $(\tilde{f}, S)$ is a $\theta$-generalized int-soft subfield over $F$.

Proposition 3.4. If $(\tilde{f}, F)$ is a $\theta$-generalized int-soft subfield of $F$, then

(1) $(\forall a \in F)(\tilde{f}(0) \supseteq \theta \cap \tilde{f}(a))$.

(2) $(\forall a \in F)(a \neq 0 \Rightarrow \tilde{f}(1) \supseteq \theta \cap \tilde{f}(a))$.

(3) $\tilde{f}(0) \supseteq \theta \cap \tilde{f}(1)$. 
Proof. (1) For all $a \in F$, we have

$$
\begin{aligned}
\tilde{f}(0) & =\tilde{f}(a+(-a)) \\
& \supseteq \theta \cap \tilde{f}(a) \cap \tilde{f}(-a) \\
& \supseteq \theta \cap \tilde{f}(a) \cap(\theta \cap \tilde{f}(a))=\theta \cap \tilde{f}(a)
\end{aligned}
$$

by using (3.1) and (3.2).

(2) is similar to the proof of (1), and (3) is by (1).

If we take $\theta=U$ in Proposition 3.4, then we have the following corollary.

Corollary $3.5([30])$. If $(\tilde{f}, F)$ is an int-soft subfield of $F$, then

(1) $(\forall a \in F)(\tilde{f}(0) \supseteq \tilde{f}(a))$.

(2) $(\forall a \in F)(a \neq 0 \Rightarrow \tilde{f}(1) \supseteq \tilde{f}(a))$.

(3) $\tilde{f}(0) \supseteq \tilde{f}(1)$.

Theorem 3.6. For a soft set $(\tilde{f}, F)$ over $F$, the following assertions are equivalent.

(1) $(\tilde{f}, F)$ is a $\theta$-generalized int-soft subfield of $F$.

(2) The nonempty $\gamma$-inclusive set

$$
i_{F}(\tilde{f} ; \gamma):=\{a \in F \mid \gamma \subseteq \tilde{f}(a)\}
$$

of $(\tilde{f}, F)$ is a subfield of $F$ for all $\gamma \in \mathscr{P}(U)$ with $\gamma \subseteq \theta$.

Proof. Assume that $(\tilde{f}, F)$ is a $\theta$-generalized int-soft subfield of $F$. Let $a, b \in$ $i_{F}(\tilde{f} ; \gamma)$ where $\gamma \in \mathscr{P}(U)$ with $\gamma \subseteq \theta$. Then $\tilde{f}(a) \supseteq \gamma$ and $\tilde{f}(b) \supseteq \gamma$. It follows that

$$
\begin{aligned}
\tilde{f}(a-b) & =\tilde{f}(a+(-b)) \\
& \supseteq \theta \cap \tilde{f}(a) \cap \tilde{f}(-b) \\
& \supseteq \theta \cap \tilde{f}(a) \cap(\theta \cap \tilde{f}(b)) \\
& \supseteq \theta \cap \gamma=\gamma .
\end{aligned}
$$


Hence $a-b \in i_{F}(\tilde{f} ; \gamma)$. Similarly, $a b \in i_{F}(\tilde{f} ; \gamma)$. If $a \neq 0$, then

$$
\tilde{f}\left(a^{-1}\right) \supseteq \theta \cap \tilde{f}(a) \supseteq \theta \cap \gamma=\gamma
$$

and so $a^{-1} \in i_{F}(\tilde{f} ; \gamma)$. Thus $i_{F}(\tilde{f} ; \gamma)$ is a subfield of $F$ for all $\gamma \in \mathscr{P}(U)$ with $\gamma \subseteq \theta$ and $i_{F}(\tilde{f} ; \gamma) \neq \emptyset$.

Conversely, suppose that (2) is valid. Let $x, y \in F$ be such that $\tilde{f}(x)=\gamma_{x}$ and $\tilde{f}(y)=\gamma_{y}$. Take $\gamma=\theta \cap \gamma_{x} \cap \gamma_{y}$. Then $x, y \in i_{F}(\tilde{f} ; \gamma)$, and so $x+y \in$ $i_{F}(\tilde{f} ; \gamma)$. Thus

$$
\tilde{f}(x+y) \supseteq \gamma=\theta \cap \gamma_{x} \cap \gamma_{y}=\theta \cap \tilde{f}(x) \cap \tilde{f}(y),
$$

which shows that (3.1) is valid. Similarly, we can prove that (3.2), (3.2) and (3.4) are valid. Therefore $(\tilde{f}, F)$ is a $\theta$-generalized int-soft subfield of $F$.

If we take $\theta=U$ in Theorem 3.6, then we have the following corollary.

Corollary 3.7 ([30]). A soft set $(\tilde{f}, F)$ over $F$ is an int-soft subfield of $F$ if and only if the nonempty $\gamma$-inclusive set

$$
i_{F}(\tilde{f} ; \gamma):=\{a \in F \mid \gamma \subseteq \tilde{f}(a)\}
$$

of $(\tilde{f}, F)$ is a subfield of $F$ for all $\gamma \in \mathscr{P}(U)$.

Definition 3.8 ([30]). Let $V$ be an algebra over $F$ and let $(\tilde{f}, F)$ be an intsoft subfield of $F$. A soft set $(\tilde{g}, V)$ is called an int-soft algebra over $(\tilde{f}, F)$ if it satisfies the following conditions:

(1) $(\forall x, y \in V)(\tilde{g}(x+y) \supseteq \tilde{g}(x) \cap \tilde{g}(y))$.

(2) $(\forall a \in F)(\forall x \in V)(\tilde{g}(a x) \supseteq \tilde{f}(a) \cap \tilde{g}(x))$.

(3) $(\forall x, y \in V)(\tilde{g}(x y) \supseteq \tilde{g}(x) \cap \tilde{g}(y))$.

(4) $(\forall x \in V)(\tilde{f}(1) \supseteq \tilde{g}(x))$.

Definition 3.9. Let $V$ be an algebra over $F$ and let $(\tilde{f}, F)$ be a $\theta$-generalized int-soft subfield of $F$. A soft set $(\tilde{g}, V)$ is called a $\theta$-generalized int-soft algebra 
over $(\tilde{f}, F)$ if there exists $\theta \in \mathscr{P}^{*}(U)$ such that

$$
\begin{aligned}
& (\forall x, y \in V)(\tilde{g}(x+y) \supseteq \theta \cap \tilde{g}(x) \cap \tilde{g}(y)) . \\
& (\forall a \in F)(\forall x \in V)(\tilde{g}(a x) \supseteq \theta \cap \tilde{f}(a) \cap \tilde{g}(x)) . \\
& (\forall x, y \in V)(\tilde{g}(x y) \supseteq \theta \cap \tilde{g}(x) \cap \tilde{g}(y)) . \\
& (\forall x \in V)(\tilde{f}(1) \supseteq \theta \cap \tilde{g}(x)) .
\end{aligned}
$$

Example 3.10. In Example $3.3(2),(\tilde{f}, F)$ is a $\theta$-generalized int-soft subfield over $F$. Then, clearly the soft set $(\tilde{g}, S)$ (where $\tilde{g}: S \longrightarrow \mathscr{P}(F)$ ) is a $\theta$ generalized int-soft algebra over $(\tilde{f}, F)$.

Proposition 3.11. Let $V$ be an algebra over $F$ and let $(\tilde{f}, F)$ be a $\theta$-generalized int-soft subfield of $F$. If $(\tilde{g}, V)$ is a $\theta$-generalized int-soft algebra over $(\tilde{f}, F)$, then $\tilde{f}(0) \supseteq \theta \cap \tilde{g}(x)$ for all $x \in V$.

Proof. For any $x \in V$, we have $\tilde{f}(0) \supseteq \theta \cap \tilde{f}(1) \supseteq \theta \cap(\theta \cap \tilde{g}(x))=\theta \cap \tilde{g}(x)$.

Corollary $3.12([30])$. Let $V$ be an algebra over $F$ and let $(\tilde{f}, F)$ be an intsoft subfield of $F$. If $(\tilde{g}, V)$ is an int-soft algebra over $(\tilde{f}, F)$, then $\tilde{f}(0) \supseteq \tilde{g}(x)$ for all $x \in V$.

Theorem 3.13. Let $V$ be an algebra over $F$ and let $(\tilde{f}, F)$ be a $\theta$-generalized int-soft subfield of $F$. Then a soft set $(\tilde{g}, V)$ is a $\theta$-generalized int-soft algebra over $(\tilde{f}, F)$ if and only if the following conditions are valid:

(1) $(\forall a, b \in F)(\forall x, y \in V)(\tilde{g}(a x+b y) \supseteq \theta \cap(\tilde{f}(a) \cap \tilde{g}(x)) \cap(\tilde{f}(b) \cap \tilde{g}(y)))$.

(2) $(\forall x, y \in V)(\tilde{g}(x y) \supseteq \theta \cap \tilde{g}(x) \cap \tilde{g}(y))$.

(3) $(\forall x \in V)(\tilde{f}(1) \supseteq \theta \cap \tilde{g}(x))$.

Proof. Assume that $(\tilde{g}, V)$ is a $\theta$-generalized int-soft algebra over $(\tilde{f}, F)$. Using (3.5) and (3.6), we have

$$
\begin{aligned}
\tilde{g}(a x+b y) & \supseteq \theta \cap \tilde{g}(a x) \cap \tilde{g}(b y) \\
& \supseteq \theta \cap(\theta \cap \tilde{f}(a) \cap \tilde{g}(x)) \cap(\theta \cap \tilde{f}(b) \cap \tilde{g}(y)) \\
& =\theta \cap(\tilde{f}(a) \cap \tilde{g}(x)) \cap(\tilde{f}(b) \cap \tilde{g}(y)) .
\end{aligned}
$$


for all $a, b \in F$ and $x, y \in V$.

(2) and (3) are by (3.7) and (3.8), respectively.

Conversely, suppose that the inclusions (1), (2) and (3) of Theorem 3.13 hold for all $a, b \in F$ and $x, y \in V$. Then

$$
\begin{aligned}
\tilde{g}(x+y) & =\tilde{g}(1 x+1 y) \supseteq \theta \cap(\tilde{f}(1) \cap \tilde{g}(x)) \cap(\tilde{f}(1) \cap \tilde{g}(y)) \\
& \supseteq \theta \cap((\theta \cap \tilde{g}(x)) \cap \tilde{g}(x)) \cap((\theta \cap \tilde{g}(y)) \cap \tilde{g}(y)) \\
& =\theta \cap \tilde{g}(x) \cap \tilde{g}(y) .
\end{aligned}
$$

The condition (3) and Proposition 3.4(3) imply that

$$
\tilde{f}(0) \supseteq \theta \cap \tilde{f}(1) \supseteq \theta \cap(\theta \cap \tilde{g}(x))=\theta \cap \tilde{g}(x)
$$

for all $x \in V$. Thus

$$
\begin{aligned}
\tilde{g}(a x) & =\tilde{g}(a x+0 x) \supseteq \theta \cap(\tilde{f}(a) \cap \tilde{g}(x)) \cap(\tilde{f}(0) \cap \tilde{g}(x)) \\
& \supseteq \theta \cap(\tilde{f}(a) \cap \tilde{g}(x)) \cap((\theta \cap \tilde{g}(x)) \cap \tilde{g}(x)) \\
& =\theta \cap \tilde{f}(a) \cap \tilde{g}(x)
\end{aligned}
$$

for all $a \in F$ and $x \in V$. Therefore $(\tilde{g}, V)$ is a $\theta$-generalized int-soft algebra over $(\tilde{f}, F)$.

Corollary $3.14([30])$. Let $V$ be an algebra over $F$ and let $(\tilde{f}, F)$ be an intsoft subfield of $F$. Then a soft set $(\tilde{g}, V)$ is an int-soft algebra over $(\tilde{f}, F)$ if and only if the following conditions are valid:

(1) $(\forall a, b \in F)(\forall x, y \in V)(\tilde{g}(a x+b y) \supseteq(\tilde{f}(a) \cap \tilde{g}(x)) \cap(\tilde{f}(b) \cap \tilde{g}(y)))$.

(2) $(\forall x, y \in V)(\tilde{g}(x y) \supseteq \tilde{g}(x) \cap \tilde{g}(y))$.

(3) $(\forall x \in V)(\tilde{f}(1) \supseteq \tilde{g}(x))$.

Theorem 3.15. Let $V$ and $W$ be algebras over F. For any algebraic homomorphism $\mu: V \rightarrow W$ and any $\theta$-generalized int-soft subfield $(\tilde{f}, F)$ of $F$, we have

(1) If $(\tilde{g}, W)$ is a $\theta$-generalized int-soft algebra over $(\tilde{f}, F)$, then the soft pre-image $\mu^{-1}(\tilde{g}, W)$ of $(\tilde{g}, W)$ under $\mu$ is also a $\theta$-generalized int-soft algebra over $(\tilde{f}, F)$. 
(2) If $(\tilde{g}, V)$ is a $\theta$-generalized int-soft algebra over $(\tilde{f}, F)$, then the soft image $\mu(\tilde{g}, V)$ of $(\tilde{g}, V)$ under $\mu$ is also a $\theta$-generalized int-soft algebra $\operatorname{over}(\tilde{f}, F)$.

Proof. (1) For any $a, b \in F$ and $x, y \in V$, we have

$$
\begin{aligned}
\mu^{-1}(\tilde{g})(a x+b y) & =\tilde{g}(\mu(a x+b y))=\tilde{g}(a \mu(x)+b \mu(y)) \\
\supseteq \theta \cap & (\tilde{f}(a) \cap \tilde{g}(\mu(x))) \cap(\tilde{f}(b) \cap \tilde{g}(\mu(y))) \\
=\theta \cap & \left(\tilde{f}(a) \cap \mu^{-1}(\tilde{g})(x)\right) \cap\left(\tilde{f}(b) \cap \mu^{-1}(\tilde{g})(y)\right), \\
\mu^{-1}(\tilde{g})(x y) & =\tilde{g}(\mu(x y))=\tilde{g}(\mu(x) \mu(y)) \\
& \supseteq \theta \cap \tilde{g}(\mu(x)) \cap \tilde{g}(\mu(y)) \\
& =\theta \cap \mu^{-1}(\tilde{g})(x) \cap \mu^{-1}(\tilde{g})(y),
\end{aligned}
$$

and $\tilde{f}(1) \supseteq \theta \cap \tilde{g}(\mu(x))=\theta \cap \mu^{-1}(\tilde{g})(x)$. Therefore, by Theorem 3.13, $\mu^{-1}(\tilde{g}, W)$ is a $\theta$-generalized int-soft algebra over $(\tilde{f}, F)$.

(2) Let $y_{1}, y_{2} \in W$. If $\mu^{-1}\left(y_{1}\right)=\emptyset$ or $\mu^{-1}\left(y_{2}\right)=\emptyset$, then

$$
\theta \cap \mu(\tilde{g})\left(y_{1}\right) \cap \mu(\tilde{g})\left(y_{2}\right)=\emptyset \subseteq \mu(\tilde{g})\left(y_{1}+y_{2}\right) .
$$

Assume that $\mu^{-1}\left(y_{1}\right) \neq \emptyset$ and $\mu^{-1}\left(y_{2}\right) \neq \emptyset$. Then $\mu^{-1}\left(y_{1}+y_{2}\right) \neq \emptyset$, and so

$$
\begin{aligned}
\mu(\tilde{g})\left(y_{1}+y_{2}\right) & =\bigcup_{x \in \mu^{-1}\left(y_{1}+y_{2}\right)} \tilde{g}(x) \supseteq \bigcup_{\substack{x_{1} \in \mu^{-1}\left(y_{1}\right) \\
x_{2} \in \mu^{-1}\left(y_{2}\right)}} \tilde{g}\left(x_{1}+x_{2}\right) \\
& \supseteq \bigcup_{\substack{x_{1} \in \mu^{-1}\left(y_{1}\right) \\
x_{2} \in \mu^{-1}\left(y_{2}\right)}}\left(\theta \cap \tilde{g}\left(x_{1}\right) \cap \tilde{g}\left(x_{2}\right)\right) \\
& =\theta \cap\left(\bigcup_{x_{1} \in \mu^{-1}\left(y_{1}\right)} \tilde{g}\left(x_{1}\right)\right) \cap\left(\bigcup_{x_{2} \in \mu^{-1}\left(y_{2}\right)} \tilde{g}\left(x_{2}\right)\right) \\
& =\theta \cap \mu(\tilde{g})\left(y_{1}\right) \cap \mu(\tilde{g})\left(y_{2}\right) .
\end{aligned}
$$


For any $y \in W$ and $a \in F$, we have

$$
\begin{aligned}
\mu(\tilde{g})(a y) & =\bigcup_{\mu(x)=a y} \tilde{g}(x)=\bigcup_{\mu(x)=y} \tilde{g}(a y) \\
& \supseteq \bigcup_{\mu(x)=y}(\theta \cap \tilde{f}(a) \cap \tilde{g}(y)) \\
& =\theta \cap \tilde{f}(a) \cap \bigcup_{\mu(x)=y} \tilde{g}(y) \\
& =\theta \cap \tilde{f}(a) \cap \mu(\tilde{g})(y) .
\end{aligned}
$$

For all $y_{1}, y_{2} \in W$, if at least one of $\mu^{-1}\left(y_{1}\right)$ and $\mu^{-1}\left(y_{1}\right)$ is empty, then the inclusion

$$
\mu(\tilde{g})\left(y_{1} y_{2}\right) \supseteq \theta \cap \mu(\tilde{g})\left(y_{1}\right) \cap \mu(\tilde{g})\left(y_{1}\right)
$$

is clear. Assume that $\mu^{-1}\left(y_{1}\right) \neq \emptyset$ and $\mu^{-1}\left(y_{2}\right) \neq \emptyset$. Then

$$
\begin{aligned}
\mu(\tilde{g})\left(y_{1} y_{2}\right) & =\bigcup_{\substack{x \in \mu^{-1}\left(y_{1} y_{2}\right)\\
}} \tilde{g}(x) \\
& =\bigcup_{\substack{x_{1} \in \mu^{-1}\left(y_{1}\right) \\
x_{2} \in \mu^{-1}\left(y_{2}\right)}} \tilde{g}\left(x_{1} x_{2}\right) \\
& \supseteq \bigcup_{\substack{x_{1} \in \mu^{-1}\left(y_{1}\right) \\
x_{2} \in \mu^{-1}\left(y_{2}\right)}}\left(\theta \cap \tilde{g}\left(x_{1}\right) \cap \tilde{g}\left(x_{2}\right)\right) \\
& =\theta \cap \bigcup_{\substack{x_{1} \in \mu^{-1}\left(y_{1}\right) \\
x_{2} \in \mu^{-1}\left(y_{2}\right)}}\left(\tilde{g}\left(x_{1}\right) \cap \tilde{g}\left(x_{2}\right)\right) \\
& =\theta \cap\left(\bigcup_{\substack{x_{1} \in \mu^{-1}\left(y_{1}\right) \\
\left(y_{1}\right)}} \tilde{g}\left(x_{1}\right)\right) \cap\left(\bigcup_{x_{2} \in \mu^{-1}\left(y_{2}\right)} \tilde{g}\left(x_{2}\right)\right) \\
& =\theta \cap \mu(\tilde{g})\left(y_{1}\right) \cap \mu(\tilde{g})\left(y_{2}\right) .
\end{aligned}
$$

Since $\tilde{f}(1) \supseteq \theta \cap \tilde{g}(x)$ for all $x \in V$, it follows that

$$
\tilde{f}(1) \supseteq \theta \cap \bigcup_{x \in \mu^{-1}(y)} \tilde{g}(x)=\theta \cap \mu(\tilde{g})(y)
$$

for all $y \in W$. Therefore $\mu(\tilde{g}, V)$ is a $\theta$-generalized int-soft algebra over $(\tilde{f}, F)$. 
Corollary 3.16 ([30]). Let $V$ and $W$ be algebras over F. For any algebraic homomorphism $\mu: V \rightarrow W$ and any int-soft subfield $(\tilde{f}, F)$ of $F$, we have

(1) If $(\tilde{g}, W)$ is an int-soft algebra over $(\tilde{f}, F)$, then the soft pre-image $\mu^{-1}(\tilde{g}, W)$ of $(\tilde{g}, W)$ under $\mu$ is also an int-soft algebra over $(\tilde{f}, F)$.

(2) If $(\tilde{g}, V)$ is an int-soft algebra over $(\tilde{f}, F)$, then the soft image $\mu(\tilde{g}, V)$ of $(\tilde{g}, V)$ under $\mu$ is also an int-soft algebra over $(\tilde{f}, F)$.

\section{Generalized int-soft hypervector spaces}

Definition $4.1([30])$. Let $V$ be a hypervector space over $F$ and $(\tilde{f}, F)$ an int-soft subfield of $F$. A soft set $(\tilde{g}, V)$ over $V$ is called an int-soft hypervector space of $V$ related to $(\tilde{f}, F)$ if the following assertions are valid:

(1) $(\forall x, y \in V)(\tilde{g}(x+y) \supseteq \tilde{g}(x) \cap \tilde{g}(y))$.

(2) $(\forall x \in V)(\tilde{g}(-x) \supseteq \tilde{g}(x))$.

(3) $(\forall a \in F)(\forall x \in V)\left(\bigcap_{y \in a \circ x} \tilde{g}(y) \supseteq \tilde{f}(a) \cap \tilde{g}(x)\right)$.

(4) $\tilde{f}(1) \supseteq \tilde{g}(\underline{0})$ where $\underline{0}$ is the zero of $(V,+)$.

Definition 4.2. Let $V$ be a hypervector space over $F$ and $(\tilde{f}, F)$ a $\theta$ generalized int-soft subfield of $F$. A soft set $(\tilde{g}, V)$ over $V$ is called a $\theta$ generalized int-soft hypervector space of $V$ related to $(\tilde{f}, F)$ if the following assertions are valid:

$$
\begin{aligned}
& (\forall x, y \in V)(\tilde{g}(x+y) \supseteq \theta \cap \tilde{g}(x) \cap \tilde{g}(y)) . \\
& (\forall x \in V)(\tilde{g}(-x) \supseteq \theta \cap \tilde{g}(x)) . \\
& (\forall a \in F)(\forall x \in V)\left(\bigcap_{y \in a \circ x} \tilde{g}(y) \supseteq \theta \cap \tilde{f}(a) \cap \tilde{g}(x)\right) . \\
& \tilde{f}(1) \supseteq \theta \cap \tilde{g}(\underline{0}) \text { where } \underline{0} \text { is the zero of }(V,+) .
\end{aligned}
$$

If $\theta=U$, then every $\theta$-generalized int-soft hypervector space is only an int-soft hypervector space. 
Example 4.3. Let us consider $F=\mathbb{Z}_{3}$ and $V=M_{2 x 2}(F)$. Also let $A=$ $\left\{\left(\begin{array}{ll}x & 0 \\ 0 & y\end{array}\right) \mid x, y \in F\right\}$.

Define an hyper operation $\circ: F \times V \longrightarrow \mathscr{P}(V)$ such that

$$
m \circ\left(\begin{array}{cc}
x & y \\
z & w
\end{array}\right)=\left(\begin{array}{cc}
0 & m y \\
m z & 0
\end{array}\right)+A
$$

for all $m \in F$ and for all $\left(\begin{array}{cc}x & y \\ z & w\end{array}\right) \in V$. Then $(V,+, \circ, F)$ is an hypervector space over $F$. Also, from Example $3.10(2),(\tilde{f}, F)$ is a $\theta$-generalized int-soft subfield over $F$. Then, clearly the soft set $(\tilde{g}, V)$ over $V$ (where $\tilde{g}: V \longrightarrow$ $\mathscr{P}(V))$ is a $\theta$-generalized int-soft hypervector space of $V$ related to $(\tilde{f}, F)$.

Proposition 4.4. Let $V$ be a hypervector space over $F$ and $(\tilde{f}, F)$ a $\theta$ generalized int-soft subfield of $F$. If $(\tilde{g}, V)$ is a $\theta$-generalized int-soft hypervector space of $V$ related to $(\tilde{f}, F)$, then

(1) $\tilde{f}(0) \supseteq \theta \cap \tilde{g}(\underline{0})$.

(2) $(\forall x \in V)(\tilde{g}(\underline{0}) \supseteq \theta \cap \tilde{g}(x))$.

(3) $(\forall x \in V)(\tilde{f}(0) \supseteq \theta \cap \tilde{g}(x))$.

Proof. It is an immediate consequence of Definition 4.2 and Proposition 3.4.

Corollary 4.5 ([30]). Let $V$ be a hypervector space over $F$ and $(\tilde{f}, F)$ an int-soft subfield of $F$. If $(\tilde{g}, V)$ is an int-soft hypervector space of $V$ related to $(\tilde{f}, F)$, then

(1) $\tilde{g}(\underline{0}) \subseteq \tilde{f}(0)$.

(2) $(\forall x \in V)(\tilde{g}(x) \subseteq \tilde{g}(\underline{0}))$.

(3) $(\forall x \in V)(\tilde{g}(x) \subseteq \tilde{f}(0))$.

Proposition 4.6. Let $V$ be a hypervector space over $F$. If $(\tilde{g}, V)$ is a $\theta$ generalized int-soft hypervector space of $V$ related to a $\theta$-generalized int-soft subfield $(\tilde{f}, F)$ of $F$, then 
(1) $(\forall x \in V)\left(\tilde{g}(x) \supseteq \theta \cap\left(\bigcap_{y \in 1 \circ x} \tilde{g}(y)\right)\right)$.

(2) $(\forall x \in V)\left(\bigcap_{y \in 1 \circ x} \tilde{g}(y) \supseteq \theta \cap \tilde{g}(x)\right)$.

Proof. (1) Let $x \in V$. Since $x \in 1 \circ x$ by (H5), we have

$$
\tilde{g}(x) \supseteq \bigcap_{y \in 1 \circ x} \tilde{g}(y) \supseteq \theta \cap\left(\bigcap_{y \in 1 \circ x} \tilde{g}(y)\right) .
$$

(2) The conditions (4.3) and (3.8) imply that

$$
\begin{aligned}
\bigcap_{y \in 1 \circ x} \tilde{g}(y) & \supseteq \theta \cap \tilde{f}(1) \cap \tilde{g}(x) \\
& \supseteq \theta \cap(\theta \cap \tilde{g}(x)) \cap \tilde{g}(x) \\
& =\theta \cap \tilde{g}(x) .
\end{aligned}
$$

for all $x \in V$.

Corollary 4.7 ([30]). Let $V$ be a hypervector space over $F$. If $(\tilde{g}, V)$ is an int-soft hypervector space of $V$ related to an int-soft subfield $(\tilde{f}, F)$ of $F$, then

$$
(\forall x \in V)\left(\tilde{g}(x)=\bigcap_{y \in 1 \circ x} \tilde{g}(y)\right) .
$$

Theorem 4.8. Assume that a hypervector space $V$ over $F$ is strongly left distributive. Let $(\tilde{f}, F)$ be a $\theta$-generalized int-soft subfield of $F$. Then a soft set $(\tilde{g}, V)$ over $V$ is a $\theta$-generalized int-soft hypervector space of $V$ related to $(\tilde{f}, F)$ if and only if the following conditions are true:

(1) $\bigcap_{z \in a \circ x+b \circ y} \tilde{g}(z) \supseteq \theta \cap(\tilde{f}(a) \cap \tilde{g}(x)) \cap(\tilde{f}(b) \cap \tilde{g}(y))$,

(2) $\theta \cap \tilde{g}(x) \subseteq \tilde{f}(1)$

for all $a, b \in F$ and all $x, y \in V$.

Proof. Assume that $(\tilde{g}, V)$ is a $\theta$-generalized int-soft hypervector space of $V$ related to $(\tilde{f}, F)$. Let $a, b \in F$ and $x, y \in V$. Then

$$
\bigcap_{z \in a \circ x+b \circ y} \tilde{g}(z)=\bigcap_{\substack{z \in u+v \\ u \in a \circ x, v \in b \circ y}} \tilde{g}(z) \supseteq \theta \cap(\tilde{f}(a) \cap \tilde{g}(x)) \cap(\tilde{f}(b) \cap \tilde{g}(y)) .
$$


Using (4.4) and Proposition 4.4(2), we have

$$
\tilde{f}(1) \supseteq \theta \cap \tilde{g}(\underline{0}) \supseteq \theta \cap \tilde{g}(x)
$$

for all $x \in V$.

Conversely suppose the conditions (1) and (2) are true. For all $x, y \in V$, we have

$$
\begin{aligned}
\tilde{g}(x+y) & \supseteq \bigcap_{z \in 1 \circ x+1 \circ y} \tilde{g}(z) \\
& \supseteq \theta \cap(\tilde{f}(1) \cap \tilde{g}(x)) \cap(\tilde{f}(1) \cap \tilde{g}(y)) \\
& \supseteq \theta \cap \tilde{g}(x) \cap \tilde{g}(y) .
\end{aligned}
$$

Since $(\tilde{f}, F)$ is a $\theta$-generalized int-soft subfield of $F$, we have

$$
\tilde{f}(0) \supseteq \theta \cap \tilde{f}(1) \supseteq \theta \cap(\theta \cap \tilde{g}(a))=\theta \cap \tilde{g}(a)
$$

and

$$
\tilde{f}(-1) \supseteq \theta \cap \tilde{f}(1) \supseteq \theta \cap(\theta \cap \tilde{g}(a))=\theta \cap \tilde{g}(a) .
$$

Note that $0 \in 0 \circ x$ for all $x \in V$. It follows that

$$
\begin{aligned}
\tilde{g}(-x) & \supseteq \bigcap_{y \in 0 \circ x+(-1) \circ x} \tilde{g}(y) \\
& \supseteq \theta \cap(\tilde{f}(0) \cap \tilde{g}(x)) \cap(\tilde{f}(-1) \cap \tilde{g}(x)) \\
& =\theta \cap \tilde{g}(x) \cap \tilde{g}(x)=\theta \cap \tilde{g}(x)
\end{aligned}
$$

for all $x \in V$. Let $a \in F$ and $x \in V$. Then

$$
\begin{aligned}
\bigcap_{y \in a \circ x} \tilde{g}(y) & \supseteq \bigcap_{\substack{y \in u+v \\
u \in 0 \circ x, v \in a \circ x}} \tilde{g}(y) \\
& \supseteq \theta \cap(\tilde{f}(0) \cap \tilde{g}(x)) \cap(\tilde{f}(a) \cap \tilde{g}(x)) \\
& \supseteq \theta \cap((\theta \cap \tilde{g}(x)) \cap \tilde{g}(x)) \cap(\tilde{f}(a) \cap \tilde{g}(x)) \\
& =\theta \cap \tilde{f}(a) \cap \tilde{g}(x) .
\end{aligned}
$$

Clearly, $\tilde{f}(1) \supseteq \theta \cap \tilde{g}(\underline{0})$. Therefore $(\tilde{g}, V)$ is a $\theta$-generalized int-soft hypervector space of $V$ related to $(\tilde{f}, F)$. 
Corollary 4.9 ([30]). Assume that a hypervector space $V$ over $F$ is strongly left distributive. Let $(\tilde{f}, F)$ be an int-soft subfield of $F$. Then a soft set $(\tilde{g}, V)$ over $V$ is an int-soft hypervector space of $V$ related to $(\tilde{f}, F)$ if and only if the following conditions are true:

(1) $\bigcap_{z \in a \circ x+b \circ y} \tilde{g}(z) \supseteq(\tilde{f}(a) \cap \tilde{g}(x)) \cap(\tilde{f}(b) \cap \tilde{g}(y))$,

(2) $\tilde{g}(x) \subseteq \tilde{f}(1)$

for all $a, b \in F$ and all $x, y \in V$.

Theorem 4.10. Let $V$ be a hypervector space over $F$ and $(\tilde{f}, F)$ a $\theta$-generalized int-soft subfield of $F$. If a soft set $(\tilde{g}, V)$ over $V$ is a $\theta$-generalized int-soft hypervector space of $V$ related to $(\tilde{f}, F)$, then the nonempty $\gamma$-inclusive set

$$
i_{V}(\tilde{g} ; \gamma):=\{x \in V \mid \gamma \subseteq \tilde{g}(x)\}
$$

of $(\tilde{g}, V)$ is a subhypervector space of $V$ over the field $i_{F}(\tilde{f} ; \gamma)$ for all $\gamma \in \mathscr{P}(U)$ with $\gamma \subseteq \theta$.

Proof. Let $x, y \in i_{V}(\tilde{g} ; \gamma)$. Then $\tilde{g}(x) \supseteq \gamma$ and $\tilde{g}(y) \supseteq \gamma$. It follows that

$$
\begin{aligned}
\tilde{g}(x-y) & =\tilde{g}(x+(-y)) \supseteq \theta \cap \tilde{g}(x) \cap \tilde{g}(-y) \\
& \supseteq \theta \cap \tilde{g}(x) \cap(\theta \cap \tilde{g}(y)) \\
& =\theta \cap \tilde{g}(x) \cap \tilde{g}(y) \supseteq \gamma .
\end{aligned}
$$

Hence $x-y \in i_{V}(\tilde{g} ; \gamma)$. Note that $i_{F}(\tilde{f} ; \gamma)$ is a subfield of $F$ (see Theorem 3.6). Let $a \in i_{F}(\tilde{f} ; \gamma), x \in i_{V}(\tilde{g} ; \gamma)$ and $y \in a \circ x$. Then

$$
\tilde{g}(y) \supseteq \bigcap_{z \in a \circ x} \tilde{g}(z) \supseteq \theta \cap \tilde{f}(a) \cap \tilde{g}(x) \supseteq \gamma,
$$

and so $y \in i_{V}(\tilde{g} ; \gamma)$ which shows that $a \circ x \subseteq i_{V}(\tilde{g} ; \gamma)$. Therefore $i_{V}(\tilde{g} ; \gamma)$ is a hypervector space over the field $i_{F}(\tilde{f} ; \gamma)$ for all $\gamma \in \mathscr{P}(U)$ with $\gamma \subseteq \theta$.

Corollary 4.11 ([30]). Let $V$ be a hypervector space over $F$ and $(\tilde{f}, F)$ an int-soft subfield of $F$. If a soft set $(\tilde{g}, V)$ over $V$ is an int-soft hypervector space of $V$ related to $(\tilde{f}, F)$, then the nonempty $\gamma$-inclusive set

$$
i_{V}(\tilde{g} ; \gamma):=\{x \in V \mid \gamma \subseteq \tilde{g}(x)\}
$$

of $(\tilde{g}, V)$ is a subhypervector space of $V$ over the field $i_{F}(\tilde{f} ; \gamma)$ for all $\gamma \in \mathscr{P}(U)$. 
Let $V$ and $W$ be hypervector spaces over $F$. A mapping $T: V \rightarrow W$ is called linear transformation (see [4]) if it satisfies:

(i) $(\forall x, y \in V)(T(x+y)=T(x)+T(y))$.

(ii) $(\forall a \in F)(\forall x \in V)(T(a \circ x) \subseteq a \circ T(x))$.

Theorem 4.12. Let $V$ and $W$ be hypervector spaces over $F$ and let $(\tilde{f}, F)$ be a $\theta$-generalized int-soft subfield of $F$. For any linear transformation $T$ : $V \rightarrow W$, if $(\tilde{g}, W)$ is a $\theta$-generalized int-soft hypervector space of $W$ related to $(\tilde{f}, F)$, then $T^{-1}(\tilde{g}, W)$ is a $\theta$-generalized int-soft hypervector space of $V$ related to $(\tilde{f}, F)$.

Proof. Let $a, b \in F$ and $x, y \in V$. Since $T$ is a linear transformation, we have

$$
\begin{aligned}
\bigcap_{z \in a \circ x+b \circ y} T^{-1}(\tilde{g})(z) & =\bigcap_{\substack{z \in a \circ x+b \circ y\\
}} \tilde{g}(T(z))=\bigcap_{u \in a \circ x, v \in b \circ y} \tilde{g}(T(u+v)) \\
& \supseteq \bigcap_{\substack{T(u) \in a \circ T(x) \\
T(v) \in b \circ T(y)}} \tilde{g}(T(u+v)) \\
& \supseteq \theta \cap(\tilde{f}(a) \cap \tilde{g}(T(x))) \bigcap(\tilde{f}(b) \cap \tilde{g}(T(y))) \\
& =\theta \cap\left(\tilde{f}(a) \cap T^{-1}(\tilde{g})(x)\right) \bigcap\left(\tilde{f}(b) \cap T^{-1}(\tilde{g})(y)\right) .
\end{aligned}
$$

Obviously, $\tilde{f}(1) \supseteq \theta \cap T^{-1}(\tilde{g})(x)$ for all $x \in V$. It follows from Theorem 4.8 that $T^{-1}(\tilde{g}, W)$ is a $\theta$-generalized int-soft hypervector space of $V$ related to $(\tilde{f}, F)$.

Corollary 4.13 ([30]). Let $V$ and $W$ be hypervector spaces over $F$ and let $(\tilde{f}, F)$ be an int-soft subfield of F. For any linear transformation $T: V \rightarrow$ $W$, if $(\tilde{g}, W)$ is an int-soft hypervector space of $W$ related to $(\tilde{f}, F)$, then $T^{-1}(\tilde{g}, W)$ is an int-soft hypervector space of $V$ related to $(\tilde{f}, F)$.

Theorem 4.14. Let $V$ and $W$ be hypervector spaces over $F$ and let $(\tilde{f}, F)$ be a $\theta$-generalized int-soft subfield of $F$. For any linear transformation $T$ : $V \rightarrow W$, if $(\tilde{g}, V)$ is a $\theta$-generalized int-soft hypervector space of $V$ related to $(\tilde{f}, F)$, then $T(\tilde{g}, V)$ is a $\theta$-generalized int-soft hypervector space of $W$ related to $(\tilde{f}, F)$. 
Proof. Let $a, b \in F$ and $x, y \in W$. If at least one of $T^{-1}(x)$ and $T^{-1}(y)$ is empty, then the inclusion

$$
\bigcap_{z \in a \circ x+b \circ y} T(\tilde{g})(z) \supseteq \theta \cap(\tilde{f}(a) \cap T(\tilde{g})(x)) \bigcap(\tilde{f}(b) \cap T(\tilde{g})(y))
$$

is clear. Assume that $T^{-1}(x)$ and $T^{-1}(y)$ are nonempty. Then there exist $u, v \in V$ such that $T(u)=x$ and $T(v)=y$. Thus

$$
\begin{aligned}
a \circ x+b \circ y & =a \circ T(u)+b \circ T(v) \\
& \supseteq T(a \circ u)+T(b \circ v) \\
& =T(a \circ u+b \circ v)
\end{aligned}
$$

since $T$ is linear. Hence $a \circ u+b \circ v \subseteq T^{-1}(a \circ x+b \circ y)$. Then

$$
\begin{aligned}
\bigcap_{w \in a \circ x+b \circ y} T(\tilde{g})(w) & =\bigcap_{\substack{w \in a \circ x+b \circ y \\
T(z)=w}} \tilde{g}(z) \\
& \supseteq \bigcap_{\substack{u \in T^{-1}(x), v \in T^{-1}(y) \\
z=z_{1}+z_{2}, z_{1} \in a \circ u, z_{2} \in b \circ v}}\left(\tilde{g}\left(z_{1}\right) \cap \tilde{g}\left(z_{2}\right)\right) \\
& \supseteq \theta \cap(\tilde{f}(a) \cap \tilde{g}(u)) \bigcap(\tilde{f}(b) \cap \tilde{g}(v)) \\
& =\theta \cap(\tilde{f}(a) \cap T(\tilde{g})(x)) \bigcap(\tilde{f}(b) \cap T(\tilde{g})(y)) .
\end{aligned}
$$

Obviously, $\tilde{f}(1) \supseteq \theta \cap T(\tilde{g})(x)$ for all $x \in W$. Therefore $T(\tilde{g}, V)$ is a $\theta$ generalized int-soft hypervector space of $W$ related to $(\tilde{f}, F)$ by Theorem 4.8 .

Corollary 4.15 ([30]). Let $V$ and $W$ be hypervector spaces over $F$ and let $(\tilde{f}, F)$ be an int-soft subfield of F. For any linear transformation $T: V \rightarrow W$, if $(\tilde{g}, V)$ is an int-soft hypervector space of $V$ related to $(\tilde{f}, F)$, then $T(\tilde{g}, V)$ is an int-soft hypervector space of $W$ related to $(\tilde{f}, F)$.

\section{Motivation and Conclusion}

Algebraic structures play an importtant role in mathematics with wide ranging applications in many disciplines such as theoretical physics, computer sciences, control engineering, information sciences, coding theory, topological 
spaces and the like. This provides sufficient motivation to researchers to review various concepts and results from the realm of abstract algebra in the broader framework of soft set theory. Soft set theory, introduced by Molodtsov [34], is an important mathematical tool to deal with uncertainties, fuzzy or vague objects and has vast applications in real life situations. Several possible applications of soft set theory in various directions are given in [34].

In this paper, we presented an application of soft set theory in an algebraic structure, called an hypervector spaces. In fact, using the notion of intersectional soft set property, we introduce the notion of $\theta$-generalized int-soft subfields, $\theta$-generalized int-soft algebras over $\theta$-generalized int-soft subfields, and $\theta$-generalized int-soft hypervector spaces. We study their properties and characterizations. In connection with linear transformations, we discusse $\theta$ generalized int-soft hypervector spaces. We hope that this work will provide a deep impact on the upcoming research in this field and other soft algebraic studies to open up new horizons of interest and innovations. Indeed, this work may serve as a foundation for further study of int-soft hypervector spaces. To extend these results, one can further study the intersectional soft hyper substructures of different algebras such as MTL-algerbas, $R_{0}$-algebras, BLalgebras, EQ-algebras, B-algebras, MV-algebras, d-algebras, Q-algebras, etc. One may also apply this concept to study some applications in many fields like decision making, knowledge base systems, data analysis, etc. Some important issues for future work are: (1) to develop strategies for obtaining more valuable results, (2) to apply these notions and results for studying related notions in other algebraic (soft) structures.

\section{Acknowledgement:}

The authors are grateful to the learned referee(s) for careful detailed reading

and helpful comments/suggestions that improved the overall presentation of this paper.

\section{References}

[1] U. Acar, F. Koyuncu and B. Tanay, Soft sets and soft rings, Comput. Math. Appl., 59 (2010), 3458-3463.

[2] A. Al-roqi, G. Muhiuddin and S. Aldhafeeri, Normal Unisoft Filters in R0-algebras, Cogent Mathematics, 1(4) (2017), 1-9.

[3] R. Ameri and O. Dehghan, Fuzzy hypervector spaces based on fuzzy singletons, Comput. Math. Appl., 61 (2011), 2933-2943. 
[4] R. Ameri and O. R. Dehghan, Fuzzy hypervector spaces, Advances in Fuzzy Systems, 2008, Article ID 295649 (2008), 9 pages.

[5] H. Aktaş and N. Çă̆man, Soft sets and soft groups, Inform. Sci., 177 (2007), 2726-2735.

[6] A. O. Atagün and A. Sezgin, Soft substructures of rings, fields and modules, Comput. Math. Appl., 61 (2011), 592-601.

[7] N. Çağman, F. Çitak and S. Enginoğlu, Soft set theory and uni-int decision making, Eur. J. Oper. Res., 207 (2010), 848-855.

[8] D. Chen, E. C. C. Tsang, D. S. Yeung and X. Wang, The parametrization reduction of soft sets and its applications, Comput. Math. Appl., 49 (2005), 757-763.

[9] P. Corsini, Prolegomena of hypergroup theory, Second edition, Aviani editor, (1993).

[10] P. Corsini and V. Leoreanu, Applications of hyperstructure theory, Kluwer Academic Publications, 2003.

[11] F. Feng, Soft rough sets applied to multicriteria group decision making, Ann. Fuzzy Math. Inform., 2 (2011), 69-80.

[12] F. Feng, Y. B. Jun and X. Zhao, Soft semirings, Comput. Math. Appl., 56 (2008), 2621-2628.

[13] G. Muhiuddin, A. M. Al-roqi and S. Aldhafeeri, Filter theory in MTLalgebras based on Uni-soft property, Bulletin of the Iranian Mathematical Society, 43(7) (2017), 2293-2306.

[14] G. Muhiuddin and A. M. Al-roqi, Unisoft Filters in R0-algebras, Journal of Computational Analysis and Applications, 19(1) (2015), 133-143.

[15] G. Muhiuddin, F. Feng and Y. B. Jun, Subalgebras of BCK/BCI-Algebras Based on Cubic Soft Sets, The Scientific World Journal, 2014, Article ID 458638 (2014), 9 pages.

[16] G. Muhiuddin, A. M. Al-roqi and S. Aldhafeeri, Filter theory in MTLalgebras based on Uni-soft property, Bulletin of the Iranian Mathematical Society, 43(7) (2017), 2293-2306.

[17] G. Muhiuddin and A. M. Al-roqi, Unisoft Filters in R0-algebras, Journal of Computational Analysis and Applications, 19(1) (2015), 133-143 . 
[18] G. Muhiuddin and A. M. Al-roqi, Cubic soft sets with applications in $B C K / B C I$-algebras, Annals of Fuzzy Mathematics and Informatics, 8(2) (2014), 291-304.

[19] G. Muhiuddin and Y. B. Jun, Sup-hesitant fuzzy subalgebras and its translations and extensions, Annals of Communications in Mathematics, 2(1) (2019), 48-56 .

[20] G. Muhiuddin and Y. B. Jun, p-semisimple neutrosophic quadruple BCIalgebras and neutrosophic quadruple p-ideals, Annals of Communication in Mathematics, 1 (1) (2018), 26-37.

[21] G. Muhiuddin, H. Harizavi and Y. B. Jun, Bipolar-valued fuzzy soft hyper $B C K$ ideals in hyper BCK algebras, Discrete Mathematics Algorithms and Applications, 12(2), 2050018 (2020), 16 pages.

[22] G. Muhiuddin, Int-soft hyper-MV-deductive systems in hyper-MV algebras, Azerbaijan Journal of Mathematics, 6(1) (2016), 39-51.

[23] Y. B. Jun and S. Z. Song and G. Muhiuddin, Hesitant fuzzy semigroups with a frontier, Journal of Intelligent and Fuzzy Systems, 30 (3) (2016), $1613-1618$.

[24] Y. B. Jun, S. Z. Song and G. Muhiuddin, Concave Soft Sets, Critical Soft Points, and Union-Soft Ideals of Ordered Semigroups, The Scientific World Journal, 2014 Article ID 467968 (2014), 11 pages.

[25] Y. B. Jun, Soft BCK/BCI-algebras, Comput. Math. Appl., 56 (2008), $1408-1413$.

[26] Y. B. Jun, K. J. Lee and A. Khan, Soft ordered semigroups, Math. Logic Q., 56 (2010), 42-50.

[27] Y. B. Jun, K. J. Lee and C. H. Park, Soft set theory applied to ideals in d-algebras, Comput. Math. Appl., 57 (2009), 367-378.

[28] Y. B. Jun, K. J. Lee and J. Zhan, Soft p-ideals of soft BCI-algebras, Comput. Math. Appl., 58 (2009), 2060-2068.

[29] Y. B. Jun and C. H. Park, Applications of soft sets in ideal theory of BCK/BCI-algebras, Inform. Sci., 178 (2008), 2466-2475.

[30] Y. B. Jun, C. H. Park and N. O. Alshehri, Hypervector spaces based on intersectional soft sets, Abstract and Applied Analysis, 2014 Article ID 784523 (2014), 6 pages. 
[31] P. K. Maji, R. Biswas and A. R. Roy, Soft set theory, Comput. Math. Appl., 45 (2003), 555-562.

[32] P. K. Maji, A. R. Roy and R. Biswas, An application of soft sets in a decision making problem, Comput. Math. Appl., 44 (2002), 1077-1083.

[33] F. Marty, Sur une generalization de la notion de groupe, in: 8th Congress des Mathematiciens Scandinaves, Stockholm, (1934), 45-49.

[34] D. Molodtsov, Soft set theory- First results, Comput. Math. Appl., 37 (1999), 19-31.

[35] C. H. Park, Y. B. Jun and M. A. Öztürk, Soft WS-algebras, Commun. Korean Math. Soc., 23 (2008), 313-324.

[36] T. Senapati, Y.B. Jun, G. Muhiuddin and K. P. Shum, Cubic intuitionistic structures applied to ideals of BCI-algebras, Analele Stiintifice ale Universitatii Ovidius Constanta-Seria Matematica, 27 (2) (2019), 213232.

[37] M. S. Tallini, Hypervector spaces, in: Fourth Int. Congress on AHA, (1990),167-174.

[38] M. S. Tallini, Weak hypervector spaces and norms in such spaces, Algebraic Hyperstructures and Applications, Hardonic Press, (1994), 199-206.

[39] T. Vougiouklis, Hyperstructures and their representations, Hardonic, Press, Inc., (1994).

[40] J. Zhan and Y. B. Jun, Soft BL-algebras based on fuzzy sets, Comput. Math. Appl., 59 (2010), 2037-2046.

G. Muhiuddin,

Department of Mathematics,

University of Tabuk,

Tabuk 71491, Saudi Arabia.

Email: chishtygm@gmail.com 